\title{
CONTRIBUTION OF WHITE DWARFS TO CLUSTER MASSES
}

\author{
TED VON HIPPEL \\ Department of Astronomy \\ University of Wisconsin \\ Madison, WI 53706, USA \\ ted@noao.edu
}

\section{Introduction}

The study of cluster white dwarfs (WDs) has been invigorated recently by the Hubble Space Telescope (HST). Recent WD studies have been motivated by the new and independent cluster distance (Renzini et al. 1996), age (von Hippel et al. 1995; Richer et al. 1997), and stellar evolution (Koester \& Reimers 1996) information that cluster WDs can provide. An important byproduct of these studies has been an estimate of the WD mass contribution in open and globular clusters. The cluster WD mass fraction is of importance for understanding the dynamical state and history of star clusters. It also bears an important connection to the WD mass fractions of the Galactic disk and halo. Current evidence indicates that the open clusters (e.g. von Hippel et al. 1996; Reid this volume) have essentially the same luminosity function (LF) as the solar neighborhood population. The case for the halo is less clear, despite the number of very good globular cluster LFs down to nearly 0.1 solar masses (e.g. Cool et al. 1996; Piotto, this volume), as the field halo LF is poorly known. For most clusters dynamical evolution should cause evaporation of the lowest mass members, biasing clusters to have flatter present-day mass functions (PDMFs) than the disk and halo field populations. Dynamical evolution should also allow cluster WDs to escape, though not in the same numbers as the much lower mass main sequence stars. The detailed connection between cluster PDMFs and the field IMF awaits elucidation from observations and the new combined N-body and stellar evolution models (Tout, this volume). Nevertheless, the WD mass fraction of clusters already provides an estimate for the WD mass fraction of the disk and halo field populations. A literature search to collect cluster WDs and a simple interpretive model follow. This is a work in progress and the full details of the literature search and the model will be published elsewhere.

\section{Observations}

Using the NASA ADS abstract service I searched the literature on white dwarfs in globular and open clusters through July 31, 1997. I included cataclysmic variables (CVs) and other types of binary systems where the authors specifically discussed the WD nature of one of the binary components. My literature search covered 82 globular cluster references and 29 open cluster references. In assessing whether an object was a cluster WD I assessed both the likelihood of cluster membership and the likelihood that the object was a WD. For the globular clusters I used relaxed criteria in both categories, allowing photometric properties to be sufficient if the reported WDs had appropriate magnitudes and colors, and if there were few, or no, field stars with the same magnitudes and colors. For the open clusters, where field contamination is much more severe, I required that the authors used proper motions or some other criteria to evaluate membership and that the resulting membership probability was at least "probable".

\section{Discussion}

At present there are reliable estimates for the total WD populations for only two clusters, the Hyades and M4. The Hyades is near enough, and its population has been well enough studied, that 
essentially all the cluster WDs have been identified. This includes 7 single WDs and 3 WDs in binaries. The combined total mass is $6.4 M_{\odot}$ (Boehm-Vitense 1993; White et al. 1993; Wegner et al. 1989). Errors on individual masses are generally $\leq 5 \%$, so the expected error of the total mass is smaller than 5\%. More importantly, it is unlikely that the current Hyades census is missing a significant number of cluster WDs (Reid, private communication). The total mass of the Hyades is $410-480 M_{\odot}$ (Reid 1992). The WD mass fraction in this young $(\sim 0.7 \mathrm{Gyr}$, Weidemann et al. 1992) cluster is thus $0.013-0.016$.

For the globular cluster M4 the situation is very different. Observations would be needed to $\mathrm{V} \geq 31$ over the entire cluster field to count all the WDs. Currently this is impossible, so Richer et al. (1995) used the number of Horizontal branch stars and their evolutionary lifetimes as compared to the lifetime of the cluster WDs (essentially the cluster age) to estimate the number of cluster WDs. They calculate that M4 contains $2 \times 10^{4}$ WDs. Among the $\geq 200$ that Richer et al. (1997) find in M4, they determine a mean mass of $0.51 \pm 0.03 M_{\odot}$. Since the observable WDs are younger than the cluster, and therefore from lower mass progenitors, I adjusted the mean cluster WD mass to $0.55 M_{\odot}$. Sigurdsson (1993) used dynamical models to estimate that the mass of M4 is $\sim 10^{5} M_{\odot}$. The WD mass fraction for this old cluster ( $\sim 12$ Gyr, e.g. Chaboyer et al. 1997) is thus $0.11 \pm 0.05$, where I have guessed that the dominant sources of uncertainty yield a precision of $50 \%$.

The WD mass fractions for the Hyades and M4 supports a picture where WDs account for $\sim 1 \%$ by mass of 1 Gyr populations and $\sim 10 \%$ by mass of 10 Gyr populations. How reasonable is such an interpretation? To address this I have created a simple model of cluster formation and evolution. I assume clusters are created in a single burst star formation event and that their mass functions can be characterized by a single power law, $N \sim M^{\alpha}$, over the mass range $0.1 \leq M / M_{\odot} \leq 80$. Current HST work on globular clusters (e.g. Piotto, this volume) is roughly consistent with the single power law model, at least below the present-day turn-off mass, $\sim 0.85 M_{\odot}$. The timescales of stellar evolution are from the parameterizations of Eggleton, Fitchett, \& Tout (1989). Evolving stars up to either 5 or $8 M_{\odot}$ (Koester \& Reimers 1996) are converted to WDs via the initial mass final mass relation as parameterized by von Hippel et al. (1997) and based on Weidemann \& Koester (1983). All gas ejected from evolving stars and all neutron star and black hole remnants are assumed to leave the cluster. These models do not include cluster binary stars. Open clusters are known to have a large number of binaries while globular clusters have binary fractions typically $\leq 5 \%$ (e.g. Richer et al. 1997). The challenge in comparing these models to the clusters is to observationally correct for binaries in the open clusters. This has been done for the Hyades. Finally, I do not model cluster kinematical evolution. Thus clusters which have undergone significant dynamical evolution should show marked differences from these simple models. Mass segregation can be simply corrected for since King models for positions near a few core radii (Cool, Piotto, \& King 1996; Piotto, this volume) have consistently shown that the PDMFs in those regions are expected to be very similar to the global MF. Most globular cluster observations have been taken at just such intermediate cluster radii. Stellar evaporation, on the other hand, would pose a problem for the models. I note, however, that globular clusters contain many stars down to at least $0.2 M_{\odot}$, so I do not expect globular clusters to have lost a significant fraction of their WDs.

Figure 1 compares the simple model to the data for M4 and the Hyades. The M4 WD mass fraction is consistent with a Salpeter IMF, while the Hyades WD mass fraction is consistent with a somewhat steeper IMF. The Hyades is likely to have seen a considerable amount of dynamical evolution (Weidemann et al. 1992), however, and it is difficult to know how to make the appropriate correction to the stellar number counts. Regardless, it appears that the M4 and Hyades WD mass fractions are reasonable, and probably typical for clusters of their age. The general picture is thus that the old stellar populations of the Galactic disk, bulge, and halo should contain $\sim 10 \%$ of their stellar mass in the form of WDs. Ultimately, precise comparisons between the field and cluster MFs for both the disk and halo would test whether the clusters we see today are typical of those which formed the field populations. 


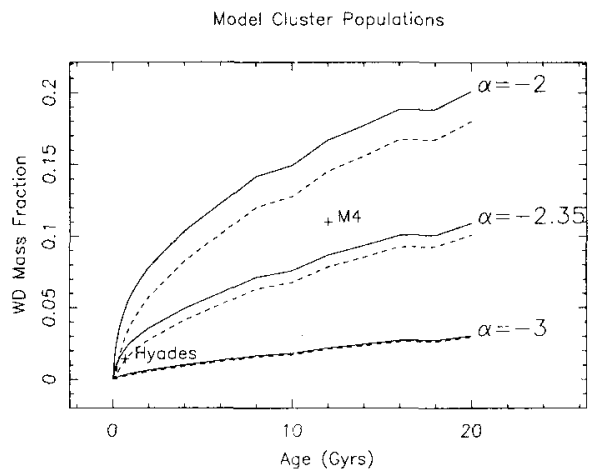

Figure 1. The WD mass fraction for the model clusters as a function of the IMF slope, for $M_{u p}=5$ (dashed lines) and 8 (solid lines) $M_{\odot}$. Also plotted are the WD mass fraction values for M4 and the Hyades.

\section{References}

Boehm-Vitense, E. 1993, AJ, 106, 1113

Chaboyer, B., Demarque, P., Kernan, P.J., \& Krauss, L.M. 1997, ApJ, in press

Cool, A.M., Piotto, G., \& King, I.R. 1996, ApJ, 468, 655

Eggleton, P.P., Fitchett, M.J., \& Tout, C.A. 1989, ApJ, 347, 998

Koester, D., \& Reimers, D. 1996, A\&A, 313,810

Reid, N. 1992, MNRAS, 257, 257

Renzini, A., et al. 1996, ApJ, 465, L23

Richer, H.B., et al. 1995, ApJ, 451, L17

Richer, H.B., et al. 1997, ApJ, 484, 741

Sigurdsson, S. 1993, ApJ, 415, L43

von Hippel, T., Bothun, G.D., \& Schommer, R.A. 1997, AJ, 114, 1154

von Hippel, T., et al. 1996, AJ, 112, 192

von Hippel, T., Gilmore, G., \& Jones, D.H.P. 1995, MNRAS, 273, L39

Weidemann, V., Jordan, S., Iben, I., \& Casertano, S. 1992, AJ, 104, 1876

Weidemann, V., \& Koester, D. 1983, A\&A, 121, 77

Wegner, G., Reid, I.N., \& McMahan, R.K. 1989, in White Dwarfs, ed. G. Wegner, (Springer-Verlag: Berlin), 378

White, S.M., Jackson, P.D., \& Kundu, M.R. 1993, AJ, 105, 563 